\title{
A purged argon pre-chamber for analytical glow discharge- time of flight mass spectrometry applications $\dagger$
}

\author{
L. Lobo, ${ }^{a}$ N. Bordel, ${ }^{b}$ R. Pereiro, ${ }^{a}$ A. Tempez, ${ }^{c}$ P. Chapon ${ }^{c}$ and A. Sanz-Medel ${ }^{* a}$ \\ Received 16th September 2010, Accepted 5th November 2010 \\ DOI: 10.1039/c0ja00160k
}

\begin{abstract}
Aiming at minimizing microleaks in the seal between the sample and the GD, a surrounding chamber which warrants a continuous isolating argon flow around the solid sample during the analysis, has been investigated for radiofrequency (rf) glow discharge (GD) coupled to time of flight mass spectrometry (TOFMS) applications. Three standard reference materials and two thin coatings were analysed comparing the influence of the purging pre-chamber in the analytical results, both in non-pulsed and pulsed rf-GD operation modes. Results show that the external argon flow seems to reduce the microleaks between the sample and the GD source since a diminished level of polyatomics appeared in the recorded mass spectra. Additionally, using the rf non-pulsed mode better signal reproducibility, less polyatomic interferences and, in some cases, higher ion signals could be achieved. In terms of in-depth profile capabilities, a faster penetration rate was observed when the pre-chamber was used and such difference was dependent on the sample composition. Comparatively, less benefit was apparent using the rf-pulsed mode as the temporal discrimination in such mode allows to separate analytical ion signals from interfering contaminants.
\end{abstract}

\section{Introduction}

Glow discharges (GDs) with either optical emission or mass spectrometry (MS) detection are nowadays well established techniques for the direct analysis of solids. Numerous applications have been described for a wide variety of materials, from conductors to insulators. ${ }^{1,2}$ Moreover, the relative recent introduction of pulsed discharges has upgraded the analytical performance of the technique as some of the problems found in the continuous power operation mode (e.g. overheating of the cathodes and unwanted thermal emissions) can be overcome. As a result, the analytical performance of the technique has been improved, particularly enhanced sensitivity in the afterglow time domain (i.e. after the end of the pulse) $)^{3,4}$ and temporal discrimination of the analyte from potential isobaric interferences have been also observed.,

For most analytical use configurations, the sample seals the GD source at ambient pressure, so the analysis can be run just after the sample is placed in the system, saving time compared to other techniques such as secondary ion mass spectrometry (SIMS) where a pre-vacuum chamber is needed prior to sample introduction in the SIMS analysis chamber. However, the lack of this latter chamber in the GD could also limit the capability of the technique for precise surface and near-surface analyses due to

\footnotetext{
${ }^{a}$ Department of Physical and Analytical Chemistry, Faculty of Chemistry, University of Oviedo, Julián Clavería 8, 33006 Oviedo, Spain. E-mail: asm@uniovi.e; Fax: +34 9851034 74; Tel: +34 985103474

${ }^{b}$ Department of Physics, Faculty of Science, University of Oviedo, 33007 Oviedo, Spain

${ }^{c}$ Horiba Jobin Yvon, Longjumeau, 91160, France

$\uparrow$ This article is part of a themed issue highlighting the latest work in the area of Glow Discharge Spectroscopy, including work presented at the International Glow Discharge Spectroscopy Symposium 2010, August 22-25, Albi, France.
}

the presence of residual air in the system and/or microleaks within the sample-GD set-up.

In fact, it is well known that the presence of $\mathrm{N}_{2}{ }^{+}, \mathrm{O}_{2}{ }^{+}$or $\mathrm{H}_{2}{ }^{+}$in argon plasmas diminishes the sputtering rate of sample materials and also modifies the observed ionization/excitation efficiency of the plasma. $^{7,8}$ Consequently, analytical results could be extremely influenced by the residual air present in the discharge, coming from adsorbed contaminants in the anode and sample surface or from possible microleaks in the sample-GD source setup. This fact complicates the analysis at very near-surface layers while worsening the accuracy and reproducibility of the results. Moreover, the presence of these species creates isobaric interferences that may lead to false identification and inaccurate quantification of a particular element. ${ }^{9}$ For instance, some studies show the effect of using ultrahigh purity argon for the determination of gas species in GD plasmas. ${ }^{10,11}$ Particularly, limits of detection between 1 and $10 \mathrm{ppm}$ can be obtained for nitrogen using radiofrequency glow discharge optical emission spectrometry using a heated metal $(\mathrm{Zr})$ getter. ${ }^{10}$ Also, part of these drawbacks could be overcome by minimising contaminants from the carrier gas or using a sacrificial material sputtered under the analysis conditions to clean the inner surface of the anode of the GD source. ${ }^{12}$ Another reported approach is the use of a low energy plasma prior to GD depth profiling to avoid the sputtering of the sample but to remove surface carbon in order to ensure cleaner conditions from the commencement of the depth profile. ${ }^{13}$ In any case such modifications do not contribute to reduce the microleaks in the sample-GD set-up. In this line, Marcus first proposed the use of an "argon bath" in a GD with optical emission detection. The system consisted on a brass cup covering the entire exterior part of the sample mount region ${ }^{14}$ where argon was introduced replacing the ambient air. A further improved quality of the plasma stability and increased signal to noise ratios were observed using this argon bath as well as better 
reproducibility. ${ }^{15}$ In this context, some commercial instrumentation, such as the Element GD-MS (Thermo), the VG GloQuad (VG Elemental) or GD90 (Mass Spectrometry Instruments), includes a vacuum chamber for the sample in order to eliminate risks of leaking between the sample itself and the GD cell.

With such practical concept, the aim of this work was to evaluate the possible analytical benefits of a chamber (prechamber) around the sample set-up to fill it with Ar for applications using radiofrequency (rf) GD (in continuous and pulsed power modes) coupled to a time of flight (TOF) mass spectrometer. Different homogeneous standard reference materials as well as layered samples have been used as model samples to study the influence of such Ar bath chamber in the analytical performance of the technique and using pulsed and non-pulsed operating modes.

\section{Experimental}

The rf-GD-TOFMS includes a rf-GD bay unit (rf generator, matching box, rf connector, refrigerator disc and sample mounting system with a pneumatic piston to press the sample against the source) from a GD Profiler HR instrument (Horiba Jobin Yvon, Longjumeau, France). The GD source is a copperbased modified Grimm-type chamber with a $4 \mathrm{~mm}$ diameter anode and a $2.5 \mathrm{~mm}$ inner diameter flow tube (EMPA, Switzerland). ${ }^{2}$ Once the ions are generated in the discharge source, they are extracted through the sampler cone $(\varnothing=0.5 \mathrm{~mm})$ and accelerated through the skimmer cone $(\varnothing=1 \mathrm{~mm})$ towards the ion lenses where the ions are focused into a $70 \mathrm{~cm}$ flight tube. The mass analyzer is an orthogonal time-of-flight mass spectrometer (Tofwerk, Switzerland, Thun) with a microchannel plate detector.

As described before, the GD can work either in pulsed mode with a frequency between $0.1 \mathrm{~Hz}$ and $10 \mathrm{kHz}$ or in non-pulsed mode using a $13.56 \mathrm{MHz}$ generator. ${ }^{2}$ The frequency and the pulse width can be selected independently. The rf power is supplied to the plasma through the backside of the sample and a refrigerating disc is used to keep the sample at low temperatures $\left(<5^{\circ} \mathrm{C}\right)$. Finally a pneumatic holder is used to press the sample against the GD ion source. Module and phase of the applied rf power were adapted to keep the reflected power to the minimum value in the non-pulsed and pulsed rf modes.

The pre-chamber consists of a plastic piece that surrounds the sample under analysis once it is placed in the GD source (see Fig. 1). It is made of high density polyethylene, with an inner diameter of $7.5 \mathrm{~cm}$ and allowing a sample up to $2.1 \mathrm{~cm}$ thick. It includes an inlet and an outlet so that a continuous argon flow

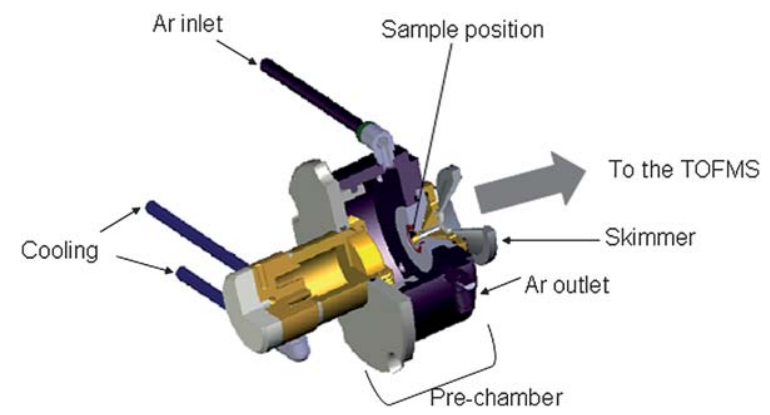

Fig. 1 Design of the Ar pre-chamber investigated.
(1.5 $\mathrm{L} \mathrm{min}^{-1}, 99.999 \%$ minimum purity) was maintained during the sample analysis.

High purity Ar (99.999\% minimum purity) from Air Liquide (Oviedo, Spain) was employed as a discharge gas for all the experiments. This gas has traces of $\mathrm{H}_{2} \mathrm{O}(\leq 3 \mathrm{ppm}), \mathrm{O}_{2}(\leq 2 \mathrm{ppm})$ and $\mathrm{C}_{n} \mathrm{H}_{m}(\leq 0.5 \mathrm{ppm})$.

Four replicate analyses were performed for each sample, with and without Ar flowing in the pre-chamber, and their results compared. The effect on the mass spectra was evaluated in nonpulsed $\mathrm{rf}$ and pulsed $\mathrm{rf}$ modes for homogeneous materials and for coated samples as well. A steel (1262b, NIST, USA), an aluminium-based (G26H2-C, MBH, England) and a zinc-based (43X-Z11, MBH) certified reference materials were used for bulk analysis experiments. The composition of the materials is collected in Table 1. In non-pulsed mode the measurements were performed at $500 \mathrm{~Pa}$ and $30 \mathrm{~W}$ using an integration time of $1 \mathrm{~s}$ (30 000 extractions). In pulsed mode, the GD conditions were $800 \mathrm{~Pa}, 100 \mathrm{~W}, 2 \mathrm{~ms}$ pulse and $4 \mathrm{~ms}$ period. One GD extraction $(33 \mu \mathrm{s})$ was employed and the different analyte intensities along the GD period were monitored. To improve the signal-to-noise ratio, an accumulation of 1400 spectra per point was used.

For depth profile analyses, comparisons of a sample consisting of a $\mathrm{Ni} / \mathrm{Cr}$ bi-layer on silicon and a $100 \mathrm{~nm}$ titanium oxide layer on steel (with and without argon flowing in the pre-chamber) were investigated in rf non-pulsed mode at the optimum depth resolution conditions, that is, $500 \mathrm{~Pa}, 20 \mathrm{~W}$ and $550 \mathrm{~Pa}, 25 \mathrm{~W}$, respectively.

\section{Results and discussion}

\section{Non-pulsed rf operation mode}

Due to the fact that the GD source is exposed to atmospheric ambient air during sample exchange between analyses, the presence of contaminant species such as nitrogen, oxygen and their related polyatomics is always observed in the MS spectra. Prior to our comparative studies with this Ar pre-chamber, an optimization of the flushing time prior to plasma ignition was performed, from 0 minutes (analysis just after sample positioning) up to 12 minutes. Results showed that beyond 3 minute purging, the level of polyatomics seems to no longer decrease. Thus, this flushing time was used for all the subsequent experiments.

Fig. $2 \mathrm{a}$ and $\mathrm{b}$ show the mass spectra obtained for the $1262 \mathrm{~b}$ sample with and without argon in the pre-chamber, respectively, at $500 \mathrm{~Pa}$ and $30 \mathrm{~W}$. As it can be seen, the use of Ar in the

Table 1 Elemental mass fraction content (\%) of major and minor investigated analytes in NIST 1262b, G26H2-C and 43X-Z11

\begin{tabular}{llll}
\hline Element & $1262 \mathrm{~b}$ & $\mathrm{G} 26 \mathrm{H} 2-\mathrm{C}$ & $43 \mathrm{X}-\mathrm{Z} 11$ \\
\hline $\mathrm{Al}$ & 0.081 & 85.51 & 11.2 \\
$\mathrm{~B}$ & 0.0025 & - & - \\
$\mathrm{Co}$ & 0.30 & - & - \\
$\mathrm{Cu}$ & 0.51 & 3.5 & 0.49 \\
$\mathrm{Fe}$ & 95.42 & 0.8 & 0.18 \\
$\mathrm{Mn}$ & 1.05 & 0.170 & 0.023 \\
$\mathrm{P}$ & 0.044 & - & - \\
$\mathrm{Pb}$ & 0.0004 & 0.200 & 0.019 \\
$\mathrm{Si}$ & 0.40 & 7.6 & $\overline{87.996}$ \\
$\mathrm{Zn}$ & - & 0.940 & \\
\hline
\end{tabular}


(a)

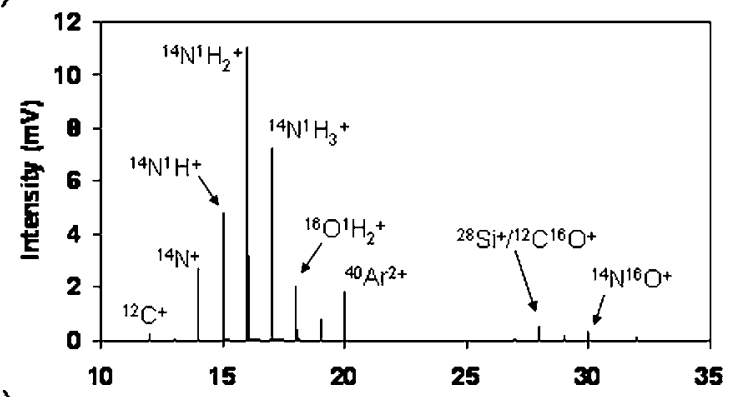

(b)

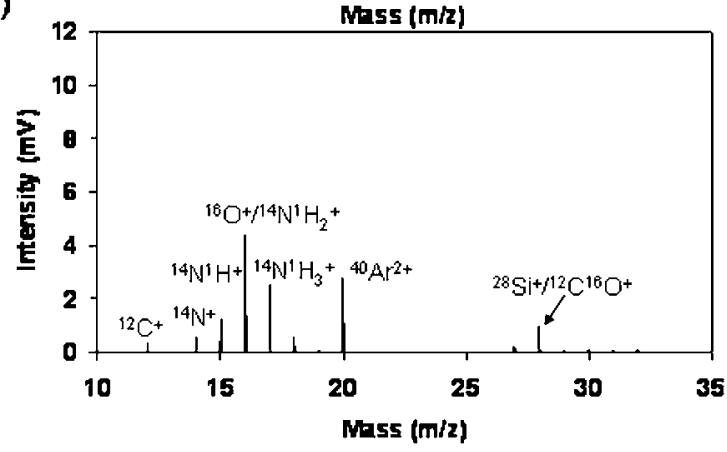

Fig. 2 Mass spectra in the interval from 10 up to $35 \mathrm{~m} / \mathrm{z}$ obtained with sample $1262 \mathrm{~b}$ at $500 \mathrm{~Pa}$ and $30 \mathrm{~W}$ (a) without Ar flowing in the prechamber and (b) with Ar flowing in the pre-chamber.

pre-chamber provides a plasma with lower contamination derived polyatomics. This can be better observed in Fig. 3 where ${ }^{14} \mathrm{~N}^{+}$ion signal intensities obtained in eight different measurements (four of them carried out with Ar flowing in the prechamber and the others without Ar in it) and using the 1262b standard are represented. As it can be seen, measurements without $\mathrm{Ar}$ showed considerably different nitrogen levels probably due to variation in the quality of the sample-source seal (which may create microleaks). On the other hand, the fact that oxygen and oxygen-species are less reduced in the presence of $\mathrm{Ar}$ in the pre-chamber could be due to the fact that nitrogen polyatomics are easily pumped down compared to water, which mainly comes from walls degassing. Conversely, flowing $\mathrm{Ar}$ inside the pre-chamber such discharge fluctuations were much reduced (i.e. argon flow seems to prevent the entrance of air in the system). Moreover, a faster stabilization of the ion signals has been also observed in this latter mode.

A similar behaviour to nitrogen was also observed for other possible contaminant species. As a result, and knowing the fact

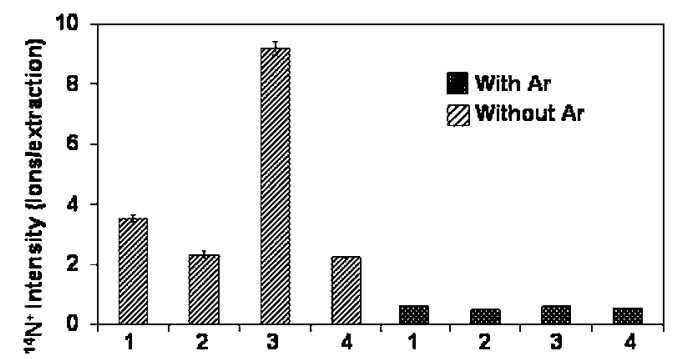

Fig. 3 Nitrogen ion signal measured in the steel $1262 \mathrm{~b}$ in continuous rf mode at $500 \mathrm{~Pa}$ and $30 \mathrm{~W}$ with and without the argon flowing in the prechamber. Numbers from 1 to 4 indicate the replicates carried out. that the presence of hydrogen, nitrogen or oxygen in the plasma results in lower sputtering rates compared to a pure $\mathrm{Ar}$ discharge, ${ }^{8,16}$ the analyte ion signals could also be affected. For example, when $1 \%$ nitrogen is added to an $\mathrm{Ar}$ plasma, the discharge resistance increases so that the number of $\mathrm{Ar}^{+}$is reduced and thus less analyte ions are formed and detected. ${ }^{17}$ Fig. 4 shows the comparative results observed with and without the argon flow in the pre-chamber for samples 1262b (Fig. 4a) and G26H2-C (Fig. 4b). It can be observed that slightly higher intensities for the analytical signals are found for the steel $1262 \mathrm{~b}$ when the $\mathrm{Ar}$ is flowing inside the pre-chamber (probably due to the lower presence of nitrogen and of other light ions such as $\mathrm{C}$, $\mathrm{H}$ and $\mathrm{O}$ in the plasma). On the other hand, for $\mathrm{G} 26 \mathrm{H} 2-\mathrm{C}$, no significant improvements in terms of sensitivity were noticed. In this latter case, the level of nitrogen was considerably lower $\left(1.3 \times 10^{4} \mathrm{cps}\right)$ compared to that measured with the sample $1262 \mathrm{~b}$ $\left(1.02 \times 10^{5} \mathrm{cps}\right)$ so less influence of the residual air on the analytical signals could be expected.

The sputtering rates in the reference materials used in this work were then measured, from two different measurements of 4 minutes, with and without the argon flow. No noticeable differences on the sputtering rates were observed between the use/no use of the argon flow for such rather long sputtering times. It is important to note here that the most remarkable effect of using Ar flowing in the pre-chamber turned out to be a clearly better signal reproducibility (see Fig. 4).

The effect of using the proposed pre-chamber on the low mass analytes signals was studied in more detail, as they are more subjected to isobaric polyatomic ions interferences. Fig. 5 shows the results obtained for two minor analytes: Fig. 5a corresponds to the analysis of ${ }^{31} \mathrm{P}^{+}$in $1262 \mathrm{~b}\left(440 \mu \mathrm{g} \mathrm{g}{ }^{-1}\right)$ and Fig. $5 \mathrm{~b}$ to ${ }^{55} \mathrm{Mn}^{+}$ in 43X-Z11 $\left(230 \mu \mathrm{g} \mathrm{g}^{-1}\right)$. As can be seen, higher ion intensities

(a)

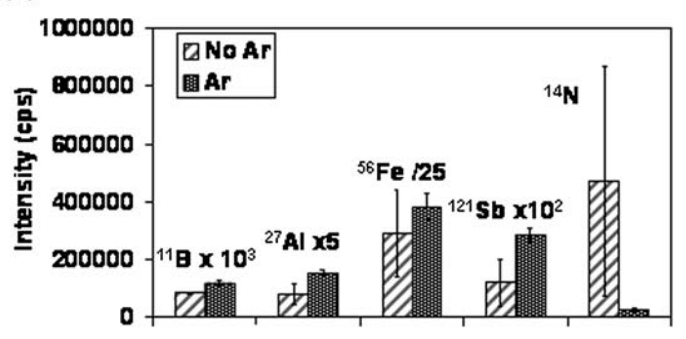

(b)

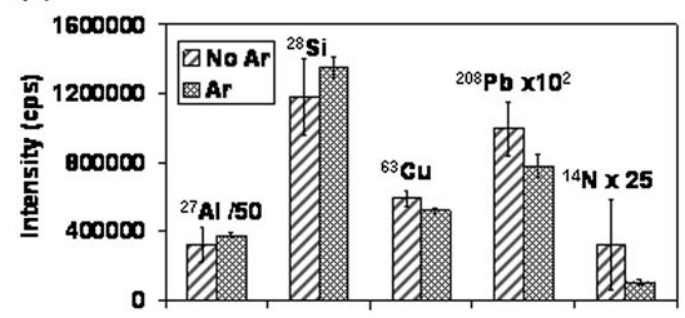

Fig. 4 Comparative average ion signals of analytes present in samples (a) 1262b, and (b) G26H2-C, obtained in continuous rf mode at $500 \mathrm{~Pa}$ and $30 \mathrm{~W}$. The averaged intensity refers to the last two minutes measurement (in order to avoid the effect on plasma stabilization). The error bar corresponds to relative standard deviation measured in four replicates. 


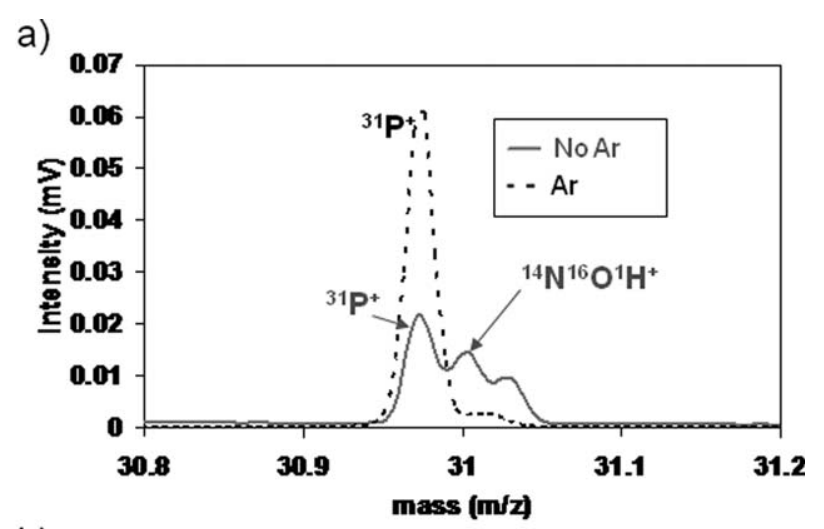

b)

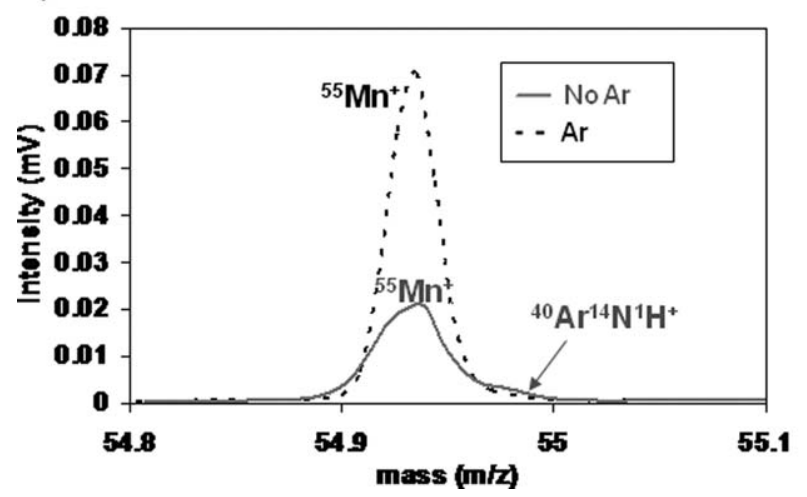

Fig. 5 Averaged mass spectrum between $\mathrm{m} / \mathrm{z} 30.8$ and 31.2 of sample $1262 \mathrm{~b}$ and between $\mathrm{m} / \mathrm{z} 54.8$ and 55.1 of sample 43X-Z11 in the absence and presence of argon in the pre-chamber during 2 minutes of sputtering: (a) ${ }^{31} \mathrm{P}^{+}$and (b) ${ }^{55} \mathrm{Mn}^{+}$. The discharge conditions were $500 \mathrm{~Pa}$ and $30 \mathrm{~W}$.

were recorded when the discharge was produced in the presence of argon in the pre-chamber. Moreover, the $\mathrm{P}^{+}$signal is clearly interfered by ${ }^{14} \mathrm{~N}^{16} \mathrm{O}^{1} \mathrm{H}^{+}$in the absence of argon in the prechamber.

These results are of particular interest for quantitative analysis, especially for the outer part layers, where a fast stabilization, reproducible signals and an as clean as possible system are essential for a proper characterization of the materials.

\section{Pulsed rf mode}

Similar studies to those carried out in rf continuous mode were performed in pulsed mode at $800 \mathrm{~Pa}, 100 \mathrm{~W}, 2 \mathrm{~ms}$ pulse and $4 \mathrm{~ms}$ period. Fig. 6 shows the pulse profile (analyte ion intensities variation along the GD period) obtained for several possible contaminants as well as ${ }^{36} \mathrm{Ar}^{+}$and ${ }^{63} \mathrm{Cu}^{+}$measured in the 43XZ11 standard, in the absence of argon in the pre-chamber (in this case, the pulse profiles obtained with and without the argon flow in the pre-chamber were rather similar). As already described, ${ }^{18}$ contaminant ions show a rapid increase in the prepeak region before decaying in the plateau. This is attributed to electron impact ionization processes of the steady state population of contaminant gas atoms present in the discharge environment and near to the exit orifice. Later on, the signal for ${ }^{63} \mathrm{Cu}^{+}$(black line in Fig. 6) appears in the pulse profile, reaching a steady state during the rf pulse. Finally, once the discharge is turned off (afterglow), an enhancement of the analyte ion signals is observed (due to the

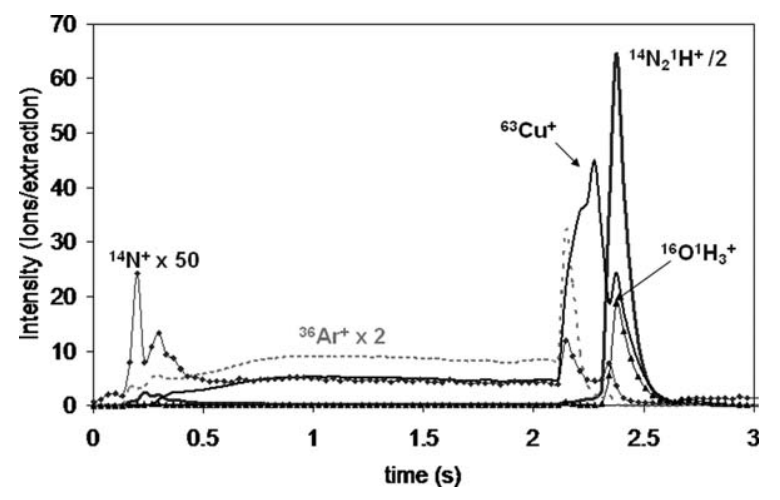

Fig. 6 Intensities of ${ }^{36} \mathrm{Ar}^{+},{ }^{14} \mathrm{~N}^{+},{ }^{14} \mathrm{~N}_{2}{ }^{1} \mathrm{H}^{+},{ }^{16} \mathrm{O}^{1} \mathrm{H}_{3}{ }^{+}$and ${ }^{63} \mathrm{Cu}^{+}$detected during a pulse time in the pulsed rf-GD mode. Sample: 43X-Z11. Discharge conditions: $100 \mathrm{~W}, 800 \mathrm{~Pa}$, pulse width: $2 \mathrm{~ms}$ and RF period: $4 \mathrm{~ms}$.

high population of Ar metastable produced from the recombination of $\mathrm{Ar}^{+}$with electrons). Also, prior to the $\mathrm{Cu}^{+}$maximum, a rapid increase of the Ar signal is detected due to the fact that argon ions are no longer lost on drift tube walls by ambipolar diffusion once the rf power is switched off. ${ }^{19}$ Moreover, this afterglow time domain offers the possibility to discriminate the analyte ion signal from possible isobaric polyatomic interferences, as interferences are formed at different times in the $\mathrm{rf}$ pulsed regime and at different spatial locations, ${ }^{20}$ in our case, the monoatomic ions are observed just right after the end of the $\mathrm{rf}$ pulse (see small signal of ${ }^{14} \mathrm{~N}^{+}$and ${ }^{63} \mathrm{Cu}^{+}$in Fig. 6) while the polyatomics appear at longer times.

In the rf pulsed mode case, the effect of using the argon prechamber was investigated in both the plateau and the afterglow regions. The prepeak was not used for data evaluation since the observed analytical signals were negligible. In this time domain, ${ }^{21}$ the sample sputtering is just starting and some microseconds are needed for the diffusion of the sputtered particles across the GD cell before they reach the sampler. Table 2 resumes the ion signals for several contaminant species measured in the plateau as well as in the afterglow. The sample employed here was the steel matrix 1262b. The intensities measured in the plateau are the result of averaging the signals detected along $1.5 \mathrm{~ms}$ of the rf pulse (steady state ion signal intensities). The intensities given in Table 2 for the afterglow region correspond to the average of three TOFMS extractions around the maximum. In the table it is indicated the delay time with respect to the end of the rf pulse at which the signal intensity has been measured for each ion. Therefore, and similarly to the results in $\mathrm{rf}$ continuous operation mode, a reduction of the possible contamination interferences is observed when argon is flowing inside the pre-chamber (in both regions studied, the plateau and the afterglow region).

On the other hand, with regard to the analytical signals detected with and without Ar flowing inside the pre-chamber, Fig. 7 shows the spectra around ${ }^{31} \mathrm{P}^{+}$at four different times during and after the rf pulse. Unlike results shown in Fig. 3a, using the rf pulsed mode, differences in terms of sensitivity were not found after the end of the rf pulse (afterglow), neither at its maximum nor at longer delay times. A similar trend was observed for other analytes in different samples. Therefore, it seems that the surrounding chamber is not influencing noticeably 
Table 2 Averaged level of contaminants found for the 1262b standard sample in the plateau (integration time: $1.5 \mathrm{~ms}$ ) and the afterglow region (integration time: $99 \mu \mathrm{s}$ ). Results correspond to the mean value of three measurements (1.43 min per analysis) at $800 \mathrm{~Pa}, 100 \mathrm{~W}, 2 \mathrm{~ms}$ pulse width and 4 ms rf period

$1262 \mathrm{~b}\left(10^{3} \mathrm{cps}\right)$

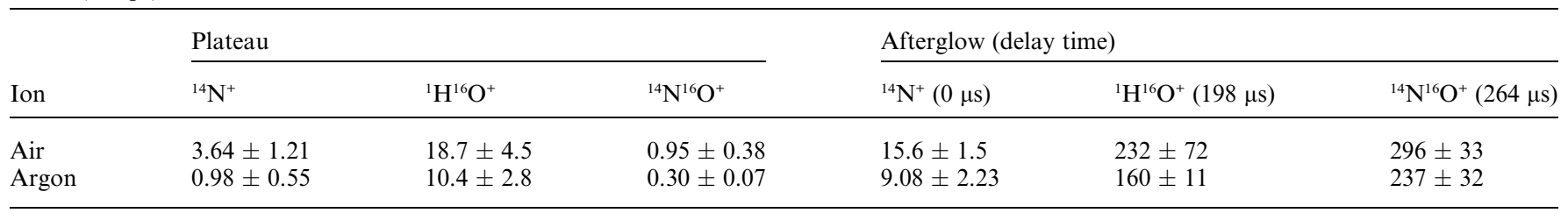

a)

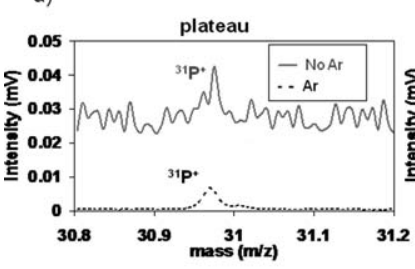

b)

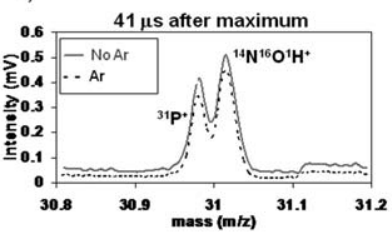

b)

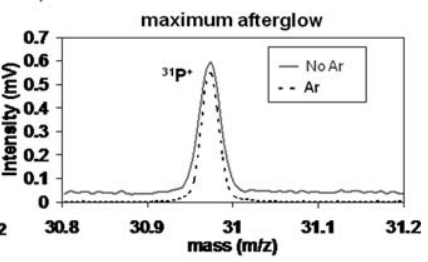

c)

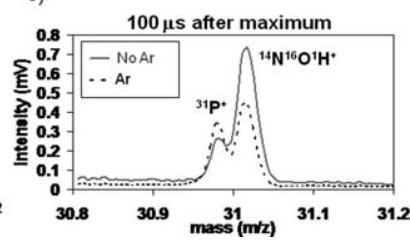

Fig. 7 Mass spectrum between $\mathrm{m} / \mathrm{z} 30.8$ and 31.2 of sample $1262 \mathrm{~b}$ measured in pulsed mode with and without argon in the pre-chamber (a) during the plateau, (b) at the maximum in the afterglow, (c and d) $41 \mu \mathrm{s}$ and $100 \mu$ s after the maximum of the afterglow. Discharge conditions were $800 \mathrm{~Pa}, 100 \mathrm{~W}, 2 \mathrm{~ms}$ pulse width and $4 \mathrm{~ms}$ rf period.

the analytical results and this could be probably attributed to the fact that the pulsed mode permits a temporal discrimination of the analyte ion signals from its corresponding polyatomic interferences. Conversely, similar to results found in rf nonpulsed mode, a better signal reproducibility was obtained by the use of the Ar pre-chamber.

\section{In-depth profile analysis}

Considering that the positive influence of using the pre-chamber was mostly observed in the rf non-pulsed mode, subsequent indepth profile experiments were focused on this latter operation mode. Fig. 8 shows the qualitative analysis of a nickel/chromium bi-layer deposited on silicon using the argon flow in the prechamber (in grey) and without argon flow (black). The experiments were carried out at optimum conditions to obtain adequate crater shapes for depth profiling (craters with flat bottoms and vertical walls, which were found to occur at $500 \mathrm{~Pa}$ and $25 \mathrm{~W}$ ). As can be seen in Fig. 8 no noticeable differences are observed in the intensities detected with and without Ar flowing in the pre-chamber. However, and in contrast to the previous experiments with the homogeneous standard materials, a faster sputtering of the analyzed sample was observed using the prechamber. Taking into account these results and the more noticeable presence of polyatomics at the very beginning of the discharge, the fact that no differences on the sputtering rates were obtained for the homogeneous materials could be attributed

to the much longer analysis time (4 minute measurements), so that the differences in the sputtering rate at the beginning of the analysis were offset by the much longer time the plasma was switched on. In the case of coating, the time needed to remove the layers is shorter, thus meaning that the pre-chamber gives rise to a "clean" plasma in a very short time. Besides, when comparing the nitrogen ion signals, a reduction was observed when using the Ar pre-chamber. Although, profiles are not shown, a reduction on the sputtering time was also observed in the rf pulsed mode when using the Ar pre-chamber at $700 \mathrm{~Pa}, 50 \mathrm{~W}, 2 \mathrm{~ms}$ rf pulse and $4 \mathrm{~ms}$ period.

Finally, a $100 \mathrm{~nm}$ titanium oxide layer on steel was also analyzed at $550 \mathrm{~Pa}, 20 \mathrm{~W}$ (see Fig. 9). In this case, a considerable difference on the sputtering rate was obtained when comparing the argon/no-argon flow results. The time needed to reach the interface was $26 \pm 3 \mathrm{~s}\left(3.8 \mathrm{~nm} \mathrm{~s}^{-1}\right)$ and $11 \pm 2 \mathrm{~s}\left(9.1 \mathrm{~nm} \mathrm{~s}^{-1}\right)$ when no $\mathrm{Ar}$ and $\mathrm{Ar}$ were flown through the pre-chamber, respectively. It appears that, once again, the reduction in nitrogen and oxygen levels, when Ar is flowing in the pre-chamber, produced an increase in the sputtering rates although no differences in the final intensities of the analytical signals were registered. These observations are of particular interest for quantitative analysis, especially in the case of very thin layers. The estimated thickness of the analyzed sample is usually calculated taking into account the sputtering rate of a series of standard reference materials but in our measurements no differences on the penetration rates have been detected when comparing the results with and without $\mathrm{Ar}$ flowing in the pre-chamber. That means that the sample thickness estimation will vary depending on the presence of such contaminants in the system, complicating accurate depth

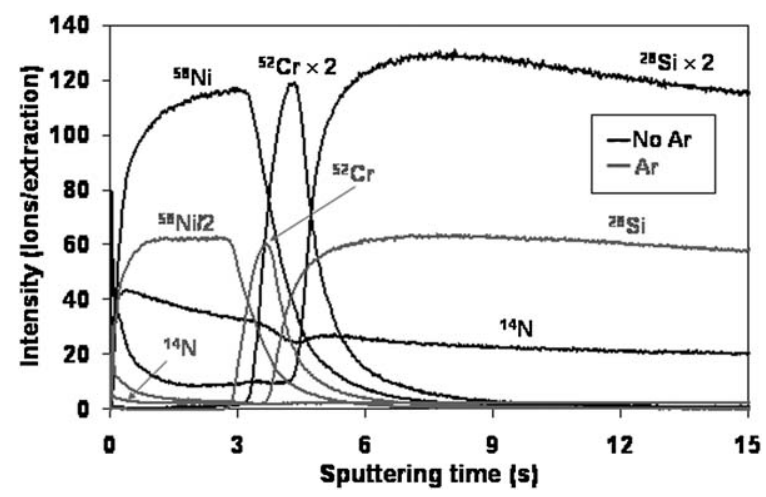

Fig. 8 Comparison of the Ni-Cr-Si depth profiles obtained without $\mathrm{Ar}$ in the pre-chamber (black line) and with argon in the pre-chamber (grey line) in continuous rf mode at $500 \mathrm{~Pa}$ and $25 \mathrm{~W}$. 

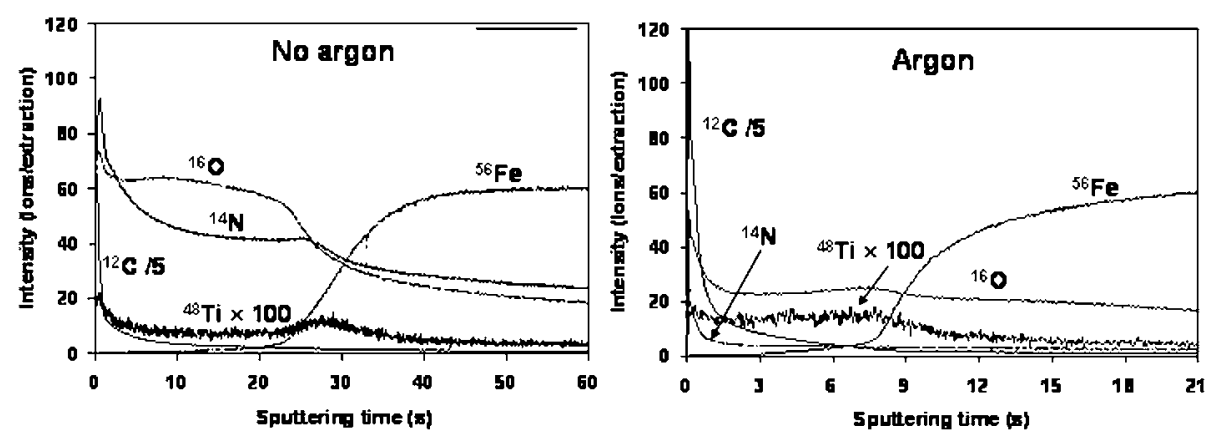

Fig. 9 In-depth profile analysis of a titanium oxide deposited on steel at $550 \mathrm{~Pa}$ and $20 \mathrm{~W}$ : (a) without argon and (b) with argon in the pre-chamber.

calculations. Moreover, the results are important for layers containing nitrogen or oxygen because inaccurate concentrations for those two elements will be found due to the significant contribution, from the leaking air contaminants, to the analytical signals coming from the sputtering of the sample.

\section{Conclusions}

The effect of using a simple and easy of use purging argon prechamber in GD-TOFMS applications has been investigated for the continuous and pulsed $\mathrm{rf}$ operating modes. A significant reduction of the ${ }^{14} \mathrm{~N}^{+},{ }^{16} \mathrm{O}^{+}$and polyatomics coming from residual air, especially of nitrogen species, was observed when the Ar pre-chamber was employed. Moreover, in the non-pulsed rf mode, the diminished level of polyatomics has proved to provide better analytical data reproducibility and, in some cases, slightly higher sensitivities.

It is important to highlight here that by using the argon flow in the pre-chamber, a fast stabilization of the plasma and a discharge less prone to contamination by air components are secured. This is crucial for in-depth profile analysis of the outermost layers where the analysis information is immediately after the GD ignition. In this line, qualitative analysis of two different coatings has been performed, showing a reduction of the time needed to reach the interface when using argon in the pre-chamber. These latter results are of particular interest for quantitative depth analysis, where the sample thickness is calculated as a function of the sputtering time of the analyzed layer.

On the other hand, slighter benefits were obtained in rf pulsed mode using the pre-chamber as compared to its influence in the rf non-pulsed mode and this can be attributed to the fact that the pulsed operation mode already allows a certain temporal discrimination between the analyte and the unwanted isobaric interferences by selecting the proper measurement time. In any case, it should be noted that the use of the pre-chamber also overcomes potential errors for thin layer thickness determination as above demonstrated for the non-pulsed mode.

Finally, the results are in good agreement (specially in rf nonpulsed mode) with those first proposed by Anfone et al. ${ }^{15}$ and performed by optical emission spectrometry, where the introduction of an argon bath not only improved the sample-tosample reproducibility, but also increased the plasma stability and signal-to-noise ratios.

\section{Acknowledgements}

Financial support from "Plan Nacional de I + D + I" (Spanish Ministry of Science and Innovation and FEDER Programme) through MAT2007-65097-C02 as well as from Consejeria de Educación y Ciencia del Principado de Asturias (ref. COF08-10, Plan I + D + I) is gratefully acknowledged.

\section{References}

1 D. Alberts, V. Vega, R. Pereiro, N. Bordel, V. M. Prida, A. Bengtson and A. Sanz-Medel, Anal. Bioanal. Chem., 2010, 396, 2833-2840.

2 M. Hohl, A. Kanzari, J. Michler, T. Nelis, K. Fuhrer and M. Gonin, Surf. Interface Anal., 2006, 38, 292-295.

3 E. P. Hastings and W. W. Harrison, J. Anal. At. Spectrom., 2004, 19, $1268-1274$.

4 A. C. Muñiz, J. Pisonero, L. Lobo, C. González, N. Bordel, A. Tempez and P. Chapon, J. Anal. At. Spectrom., 2008, 23, 12391246.

5 W. Hang, C. Baker, B. W. Smith, J. D. Winefordner and W. W. Harrison, J. Anal. At. Spectrom., 1997, 12, 143-149.

6 L. Lobo, J. Pisonero, N. Bordel, R. Pereiro, A. Tempez, P. Chapon, J. Michler, M. Hohl and A. Sanz-Medel, J. Anal. At. Spectrom., 2009, 24, 1373-1381.

7 L. Lobo, B. Fernandez, R. Pereiro, N. Bordel and A. Sanz-Medel, Anal. Bioanal. Chem., 2007, 389, 743-752.

8 B. Fernández, N. Bordel, R. Pereiro and A. Sanz-Medel, J. Anal. At. Spectrom., 2003, 18, 151-156.

9 A. Bengtson, J. Anal. At. Spectrom., 2003, 18, 1066-1068.

10 M. L. Hartenstein and R. K. Marcus, J. Anal. At. Spectrom., 1997, 12, $1027-1032$.

11 R. E. Valiga, D. C. Duckworth and D. H. Smith, Rapid Commun. Mass Spectrom., 1996, 10, 305-310.

12 D. Klemm, M. Stangl, A. Peeva, V. Hoffmann, K. Wetziga and J. Eckert, Surf. Interface Anal., 2008, 40, 418-422.

13 I. S. Molchan, G. E. Thompson, P. Skeldon, N. Trigoulet, P. Chapon, A. Tempez, J. Malherbe, L. Lobo Revilla, N. Bordel, Ph. Belenguer, T. Nelis, A. Zahri, L. Therese, Ph. Guillot, M. Ganciu, J. Michler and M. Hohl, J. Anal. At. Spectrom., 2009, 24, 734-741.

14 R. K. Marcus, Winter Conference of Plasma Spectrochemistry, Fort Lauderdale, FL, 1996.

15 A. B. Anfone and R. K. Marcus, J. Anal. At. Spectrom., 2001, 16, 506-513.

16 A. Menéndez, J. Pisonero, R. Pereiro, N. Bordel and A. Sanz-Medel, J. Anal. At. Spectrom., 2003, 18, 557-563.

17 G. P. Jackson and F. L. King, Spectrochim. Acta, Part B, 2008, 58, 185-209.

18 J. A. Klingler, C. M. Barshick and W. W. Harrison, Anal. Chem., 1991, 63, 2571-2576.

19 A. Tempez, N. Bordel, M. Hohl, L. Lobo, J. Orphal, C. Diplasu, A. Surmeian, M. Ganciu, T. Nakamura, Proceedings of the $18^{\text {th }}$ International Symposium on Plasma Chemistry, ed. K. Tachibana, Kyoto, Japan, ISBN 978-4-9903773-1-1, 2007, pp. 267-271.

20 R. E. Steiner, C. L. Lewis and V. Majidi, J. Anal. At. Spectrom., 1999, 14, $1537-1541$.

21 C. Pan and F. L. King, J. Am. Soc. Mass Spectrom., 1993, 4, 724-732. 